\title{
Evaluation of quality and readability of online patient information on osteoporosis and osteoporosis drug treatment and recommendations for improvement
}

\author{
F. Crawford-Manning ${ }^{1} \cdot$ C. Greenall ${ }^{2} \cdot$ A. Hawarden ${ }^{2}$ (D) $\cdot$ L. Bullock $^{2}$ (D) S. Leyland ${ }^{3} \cdot$ C. Jinks $^{2}$ (D) J. Protheroe ${ }^{2}$ (D) \\ Z. Paskins ${ }^{1}$ (1)
}

Received: 13 September 2020 / Accepted: 14 December 2020 / Published online: 27 January 2021

(C) Crown 2021

\begin{abstract}
Summary Patient information is important to help patients fully participate in their healthcare. Commonly accessed osteoporosis patient information resources were identified and assessed for readability, quality, accuracy and consistency. Resources contained inconsistencies and scored low when assessed for quality and readability. We recommend optimal language and identify information gaps to address.

Introduction The purpose of this paper is to identify commonly accessed patient information resources about osteoporosis and osteoporosis drug treatment, appraise the quality and make recommendations for improvement.

Methods Patient information resources were purposively sampled and text extracted. Data extracts underwent assessment of readability (Flesch Reading Ease and Flesch-Kincaid Grade Level) and quality (modified International Patient Decision Aid Standards (mIPDAS)). A thematic analysis was conducted, and keywords and phrases were used to describe osteoporosis and its treatment identified. Findings were presented to a stakeholder group who identified inaccuracies and contradictions and discussed optimal language.

Results Nine patient information resources were selected, including webpages, a video and booklets (available online), from government, charity and private healthcare providers. No resource met acceptable readability scores for both measures of osteoporosis information and drug information. Quality scores from the modified IPDAS ranged from 21 to 64\% (7-21/33). Thematic analysis was informed by Leventhal's Common-Sense Model of Disease. Thirteen subthemes relating to the identity, causes, timeline, consequences and controllability of osteoporosis were identified. Phrases and words from 9 subthemes were presented to the stakeholder group who identified a predominance of medical technical language, misleading terms about osteoporotic bone and treatment benefits, and contradictions about symptoms. They recommended key descriptors for providers to use to describe osteoporosis and treatment benefits.

Conclusions This study found that commonly accessed patient information resources about osteoporosis have highly variable quality, scored poorly on readability assessments and contained inconsistencies and inaccuracies. We produced practical recommendations for information providers to support improvements in understanding, relevance, balance and bias, and to address information gaps.
\end{abstract}

Keywords Osteoporosis $\cdot$ Patient information $\cdot$ Quality $\cdot$ Readability $\cdot$ Recommendations

F. Crawford-Manning f.manning@keele.ac.uk

1 School of Medicine, Keele University \& Haywood Academic Rheumatology Centre, Stoke-on-Trent, UK

2 School of Medicine, Keele University, Newcastle-under-Lyme, Staffordshire, UK

3 Royal Osteoporosis Society, Bath, UK

\section{Introduction}

Government policy in the UK places emphasis on providing patients with good quality health information to encourage patient participation in their healthcare, by supporting the involvement of patients in their care and treatment decisionmaking processes [1]. Improving access to quality information is rated as a top research priority for people living with osteoporosis [2]. The internet is increasingly becoming an influential source of health care information [3], with as many as 
$61 \%$ of UK patients accessing medical information via the web [4]. A wide range of providers may offer patient information resource online, with government, charity and private healthcare stakeholders being perceived as more credible than other sources [5]; however, the quality of these resources, in the case of osteoporosis, is unknown.

The quality of patient information encompasses a range of issues including accuracy and comprehensiveness, accessibility, acceptability, presentation style, comprehensibility, relevance and utility and attention to health literacy [6]. Comprehensiveness relates to the appropriate range of issues that need to be covered [7]; it is important that patient information presents an accurate, comprehensive coverage of a subject for example, discussing benefits and harms of treatment in equal detail, in order to give a balanced account [8]. The current 'osteoporosis crisis', characterised by poor treatment uptake and the consequent levels of preventable fracture has been blamed on health professionals' failure to clearly communicate these aspects of osteoporosis drug treatment to patients [9].

The ease with which patient information is understandable or comprehensible will be influenced by a range of factors such as the way information is presented, the language used and the health literacy of the reader. Health literacy is defined as the degree to which individuals have the capacity to obtain, process and understand basic health information and services needed to make appropriate health decisions [10]. Health literacy includes a range of skills such as numeracy and reading; in a study assessing the readability of 345 patient information booklets available in general practices, it was found that only $24 \%$ were written at the recommended reading level for the general population (Felsch Kincaid Grade 6, equivalent to reading age 10-11) [8,11]. Furthermore, a sample of $64 \mathrm{UK}$ health information sources were found to be too complex (based on assessment by the English National Qualifications Framework), for $43 \%$ of the population to understand [9]. This rose to $61 \%$ if the information required numeracy skills in addition to interpretation of text [12]. This mismatch between complexity of information and health literacy impacts on access to healthcare and affects health outcomes. In osteoporosis, the language used to describe the condition is likely to be important; the term osteoporosis itself is often misunderstood and a systematic review of patient information needs reported uncertainty and confusion in areas relating to the nature of the condition, medication and controllability [13]. The review further suggested the need for research into optimum explanations of osteoporosis and the promotion of clear messages that avoid ambiguity [13].

The 'Improving uptake of Fracture Prevention drug treatments (iFraP) study' aims to address some of these issues, by developing a decision tool, training package and information resources to ensure consistent, accurate, understandable information provision relating to osteoporosis and its drug treatment. Our aim for this study was to inform the iFraP intervention, by identifying commonly accessed patient information resources, and within these resources, identifying frequently used phrases and descriptors using a descriptive thematic analysis [14]. Additionally, we aimed to describe the readability and quality of these resources. We sought stakeholder views on quality (including accuracy and language used) to make recommendations for improvement.

\section{Methods}

An overview of the methods is shown in Fig. 1.

\section{Identification}

We aimed to identify a purposive sample that would include sufficient diversity, would be large enough for meaningful analysis, and that would represent the majority of resources used in primary and secondary care. Our purposive sampling approach was informed by the methods of previous research investigating content of patient information booklets in osteoarthritis [15]. Patient information resources were selected on the basis that they were easily available either on the internet, through patient organisations or in osteoporosis clinics. We included webpages, booklets and videos, if transcripts were available, for analysis. First, two clinical authors (ZP, Consultant Rheumatologist and Clinical Lead for osteoporosis service and AH, Specialist Registrar in Rheumatology) identified providers and resources of patient information commonly recommended to patients in primary and secondary care that were expected to be identified in the search (informed by the methods of Misra et al. [16]). A google search using the term 'osteoporosis' was conducted on 20th December 2019 with hits being screened for UK specific patient information. Inclusion was dependent on resources being from three different types of provider: healthcare providers; charitable, voluntary or patient organisations; and the medical profession. Hits were reviewed sequentially until we had representation from all three types of provider and the inclusion of providers/resources preidentified by clinical authors [16].

\section{Data extraction}

In line with our study aims, we focused our investigation on information related to describing the condition and drug treatment. For each resource selected, text was extracted into Microsoft $®$ Word documents. Video transcripts and booklets were extracted in their entirety. Text from webpages was extracted from sections on the webpage relating to descriptions of osteoporosis and descriptions of osteoporosis drug treatment. Text relating to lifestyle management and fracture management was not included, as they were deemed outside of the scope of 


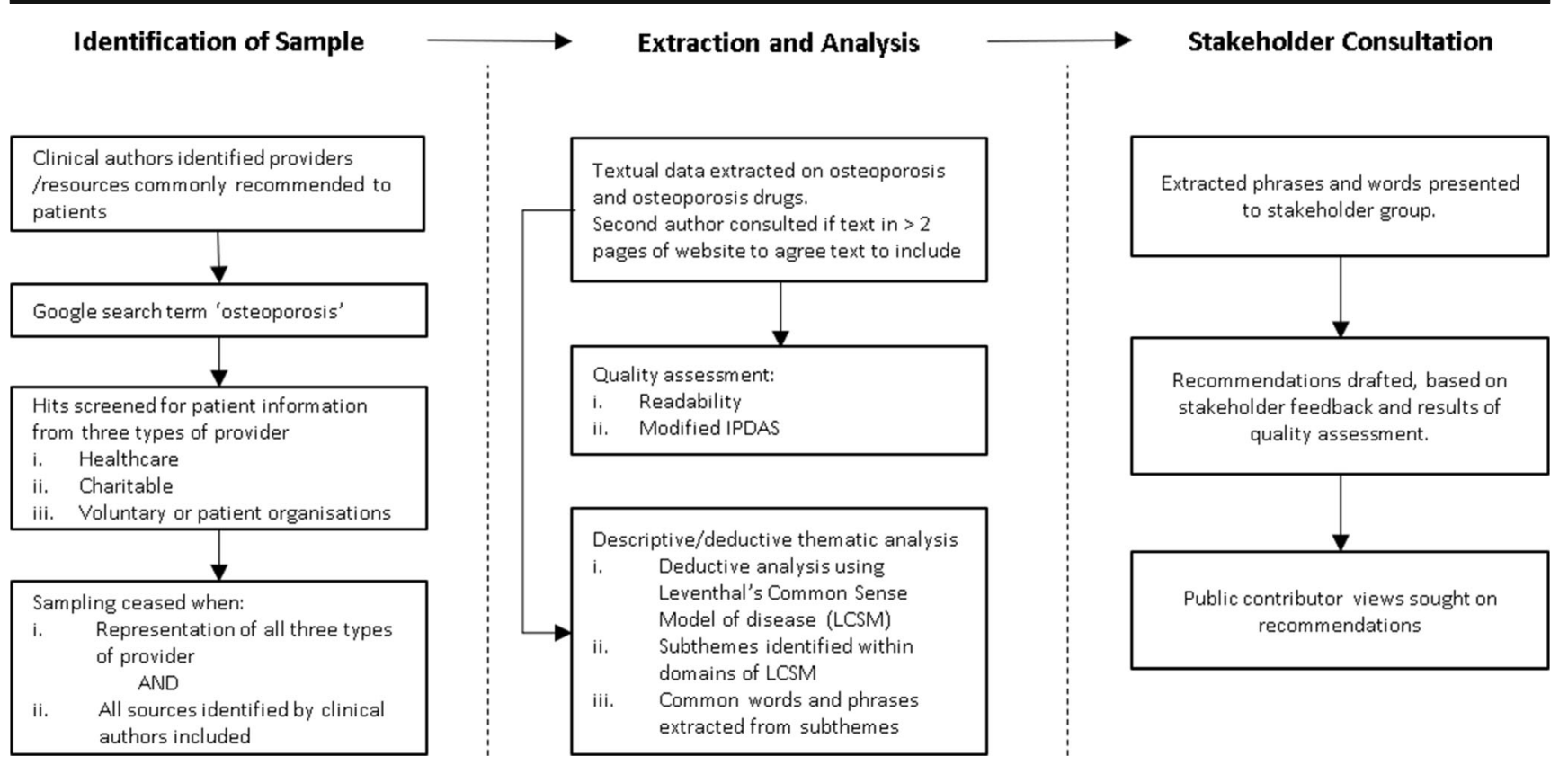

Fig. 1 Overview of methods

this study. Where websites contained information on osteoporosis drugs and the condition in more than two webpages, two authors (ZP and FCM) agreed which text to include through discussion and recorded the decisions made on inclusion.

\section{Quality assessment}

Quality assessment comprised a readability score and a quality checklist derived from patient information quality standards.

The readability scores were calculated utilising the Flesch Reading Ease (FRE) and Flesch-Kincaid Grade Level (FKGL) 'Readability' function in Microsoft@ Word 2007. Together, these scores provide a comprehensive picture of patient information readability, in line with previous methodology [8]. The FRE assesses text on a 100-point scale [17], with a high score indicating the document is easier to read. The FKGL assesses the text based on the US school grade level [18]. In contrast to the FRE, the lower the FKGL score, the easier it is to understand.

Readability was calculated in Microsoft ${ }^{\circledR}$ Word for sections on osteoporosis and osteoporosis drug management separately. Readability of drug management was separated as it has been shown previously to be more complex than descriptive, condition-specific information [19]. Readability was calculated from an average of three sections of text, each 100 words, following the methods of Protheroe et al. [8]. Sections of text were taken from the start, middle and end of extracted text (avoiding contact details). The threshold of acceptable readability was set at a FRE score of $>60$ and FKGL of $<6$ (suggesting a reading age of approximately 11 ), based on the 2011 Skills for Life Survey and the recommended level for provision of medical information [11].
In order to evaluate the quality of patient information, we utilised a modified version of the International Patient Decision Aid Standards (m-IPDAS). This scale has been modified to measure the quality of patient information resources, mapped against information quality standards attending to issues of balance and bias [20]. The m-IPDAS scale determines the quality based on eight areas: structure and layout, statement of aims, information reliability, information about treatment options, support provided to patients to make appropriate decisions, disclosures of conflicts of interest, accurate information and probabilities of treatment outcomes [20]. We included one additional question relating to the balance of presentation of benefits and risks, which was derived from an IPDAS update in 2009 [21]. In line with our study aim, we considered treatment options as drug treatments for the purpose of this exercise. An instruction sheet for completing the m-IPDAS was formulated to clarify the questions and was piloted with three reviews (FCM and $\mathrm{LB}$ or $\mathrm{CG}$ ). The $\mathrm{m}-$ IPDAS and instruction sheet are available in the supplementary materials (Supplementary Tables 1 and 2 respectively). Pairs of researchers (FCM and LB or CG) assessed data extracted on the condition of osteoporosis and osteoporosis drug treatments for each resource against the m-IPDAS criteria independently and referred to the original resource if required. Disagreements were resolved through a fourth reviewer (ZP). A total score out of 33 and a percentage was then calculated.

\section{Descriptive/deductive thematic analysis}

The extracted data was initially deductively coded; deductive coding means that a predefined list of codes are assigned, as opposed to inductive coding where themes 
emerge from the data [14]. The five domains of Leventhal's Common-Sense Model of Disease were used as a deductive coding framework [22] to ensure that analysis was theoretically driven by the domains of illness perceptions which influence coping strategies, and health behaviours, including adherence to medication [23]. The five domains are identity - the label or name given to the condition and associated symptoms; cause - individualistic ideas about the apparent cause of the condition; timeline-beliefs about how long the condition might last; consequences - beliefs about the consequences of the condition and physical, mental and social sequelae; controllability-beliefs about whether the condition can be cured or kept under control and the degree to which the individual plays a part in achieving this. The use of this model as a coding framework provides analytical focus on the description of osteoporosis in the identified patient information resources, reflecting the research question [24]. An additional round of inductive coding (meaning that codes identified from the data, rather than a pre-existing coding framework [14]) was conducted to identify subthemes within each domain. In total, the extracted data underwent three rounds of coding by two independent authors (CG or FCM, both novice qualitative researchers but with clinical knowledge of osteoporosis); after each round, coding was discussed with $\mathrm{ZP}$, an experienced qualitative researcher, and refined. ZP and FCM then agreed a final coding framework which was systematically reapplied across the extracted data by FCM and ZP.

\section{Stakeholder views and generation of recommendations}

Expert members of the stakeholder group were recruited through personal networks and the Royal Osteoporosis Society (ROS), whilst patient members were recruited through the Keele University School of Primary, Community and Social Care research users group and included $(n=12,3$ males and 9 females):

- Two ROS representatives

- Two GPs (one with health literacy expertise)

- Two hospital doctor specialists representing metabolic bone, and rheumatology

- Two osteoporosis specialist nurses

- One physiotherapist

- Two patients with osteoporosis

- One patient and public involvement and engagement (PPIE) support worker

An additional six members of the iFraP study team were also present. Stakeholders were presented with the information providers, and extracted phrases and words from each of the five domains were presented to a stakeholder group to identify any inaccurate or contradictory terms/advice and discuss optimal language, with the aim to develop recommendations for describing osteoporosis and its drug treatment. Recommendations for improving patient information were drafted by three authors, based on findings from the quality assessment and stakeholder comments and reviewed by public contributors.

\section{Results}

Nine patient information resources were identified from 6 providers: the NHS (a webpage and video) $[25,26]$; three charities (ROS (formally known as the National Osteoporosis Society), Versus Arthritis (VA) (formally known as Arthritis Research UK) and Age UK-three webpages and two pdf booklets) [27-31]; one doctor-led webpage (Patient.info) [32] and one private healthcare provider webpage (BUPA) [33]. The sample size of nine was felt to be adequate by authors and stakeholders, representing the majority of UK information providers in primary and secondary care, and being similar to previous studies in this area $[15,16]$.

Two resources contained all information on osteoporosis and osteoporosis drugs on a single webpage, and this webpage was extracted in entirety (BUPA and Age UK) [31, 33]. Three websites had a main 'osteoporosis' webpage, with a second linked webpage containing drug treatment information, both of which were extracted in entirety (NHS webpage, VA webpage and Patient.info) [26, 27, 32]. One website (ROS) [30] contained relevant information on more than two webpages; details of decision-making on information to extract for this site are available in supplementary information Table 3. Video transcripts and booklets were extracted in their entirety. A summary of the included resources is shown in Table 1 .

\section{Quality: readability}

Readability results are summarised in Table 2. Five of the nine resources included less than 300 words relating to osteoporotic drugs therefore it was only possible to calculate a single readability score rather than a mean of 3 areas. None of the resources scored within the acceptable range on both the FRE and FKGL scales ( $>60$ and $<6$ respectively). The information on osteoporosis on the ROS webpage met an acceptable FRE score, and the transcript of the information on osteoporosis drugs from the NHS video met an acceptable FRE score. No other resources met any criteria for readability for information on the condition osteoporosis or osteoporosis drug treatment. 
Table 1 Summary of included patient information resources

\begin{tabular}{|c|c|c|c|c|}
\hline Publisher & Media & Provider type & Title & Word count ${ }^{*}$ \\
\hline Age UK [31] & Webpage & Charity & Osteoporosis & 1058 \\
\hline Arthritis Research UK (ARUK) [28] & Leaflet & Charity & Osteoporosis & 6661 \\
\hline BUPA [33] & Webpage & Private sector & Osteoporosis & 3067 \\
\hline National Health Service (NHS) [26] & Webpage & Healthcare & Osteoporosis & 6265 \\
\hline NHS [25] & Video & Healthcare & Osteoporosis Video & 718 \\
\hline National Osteoporosis Society (NOS) [29] & Leaflet & Charity & All about Osteoporosis and Bone health & 23,612 \\
\hline Patient-info [32] & Webpage & Doctor led & Osteoporosis & 5202 \\
\hline Royal Osteoporosis Society (ROS) ${ }^{\dagger}[30]$ & Webpage & Charity & Understanding Osteoporosis & 6913 \\
\hline Versus Arthritis (VA) ${ }^{\ddagger}[27]$ & Webpage & Charity & Osteoporosis & 3484 \\
\hline
\end{tabular}

${ }^{*}$ Word count of extracted data

${ }^{\dagger}$ Formally National Osteoporosis Society

ॠFormally Arthritis Research UK

\section{Quality: m-IPDAS checklist assessment}

The total m-IPDAS scores of individual resources ranged from 21.2 to $63.6 \%$ and are detailed in Table 3. The highest scoring resource was the National Osteoporosis Society (NOS) booklet [29] scoring 21/33 (63.6\%), followed by the BUPA webpage [33] and Arthritis Research UK (ARUK) booklet [28], each scoring 20/33 (60.6\%). The NHS video [25] scored the lowest (7/33 (21.2\%)) and Age UK [31] second lowest $(13 / 33(39.4 \%))$.

The domains with the lowest scores were accuracy, probability, decision-making and conflicts of interest. Only 2/9 (22\%) reported the source of the evidence quoted, and no resource discussed event rates or probabilities when discussing osteoporosis drug benefits and harms. Few resources $(3 / 9,33 \%)$ recognised explicitly that the reader might be making a decision about drug treatment, and only $2 / 9$ (22\%) helped the reader to identify the physical, social and psychological consequences of untreated osteoporosis to aid in this process, while only one included content to help the reader think about their priorities and motivations. Four resources provided information on the authors of the information, and neither of the two privately owned resources stated their funding source. Scores were highest in the domains relating to content, usability and unbiased, structure and layout and reliability, although these domains all included individual items which scored low. For example, while most resources included an explanation of purpose and content, none

\begin{tabular}{llllll}
\hline Resource & \multicolumn{2}{l}{$\begin{array}{l}\text { Readability of information describing } \\
\text { osteoporosis }\end{array}$} & & \multicolumn{2}{l}{$\begin{array}{l}\text { Readability of information describing } \\
\text { osteoporosis drugs }\end{array}$} \\
\cline { 2 - 3 } \cline { 5 - 6 } & FRE mean (range) & FKGL mean (range) & & FRE mean (range) & FKGL mean (range) \\
\hline Age UK-W & $59.13(44.0-69.2)$ & $8.27(6.5-9.4)$ & & $34.1^{\dagger}$ & $11.2^{\dagger}$ \\
ARUK-L & $57.27(53.4-60.6)$ & $8.80(7.9-9.9)$ & & $55.80(45.6-70.7)$ & $9.10(6.7-11.0)$ \\
BUPA-W & $53.57(48.0-63.8)$ & $9.33(8.4-10.2)$ & & $45.9^{\dagger}$ & $9.6^{\dagger}$ \\
NHS-W & $56.53(47.8-61.6)$ & $9.30(7.8-10.4)$ & & $50.00(44.7-57.6)$ & $9.53(8.2-10.8)$ \\
NHS-V & $55.37(51.7-60.7)$ & $9.57(7.7-10.7)$ & & $68.9^{*}$ & $6.3^{\dagger}$ \\
NOS-L & $48.20(31.4-57.4)$ & $12.80(10.3-17.2)$ & & $52.13(34.7-73.1)$ & $10.93(6.1-15.4)$ \\
Patient-info-W & $57.03(50.6-64.9)$ & $8.20(7.0-9.0)$ & & $51.97(48.2-58.3)$ & $9.73(8.6-10.8)$ \\
ROS-W & $62.07(49.7-71.8)$ & $8.00(7.1-9.6)$ & & $57.9^{\dagger}$ & $8.4^{\dagger}$ \\
VA-W & $50.97(43.4-58.8)$ & $10.80(9.3-11.6)$ & & $50.1^{\dagger}$ & $10.5^{\dagger}$ \\
\hline
\end{tabular}

* Indicates a score within acceptable limits (FRE $>60$, FKGL $<6$ )

${ }^{\dagger}$ Indicates result from single assessment, not mean, due to insufficient text for 3 assessments.

W- Webpage, L- Leaflet, V-Video 


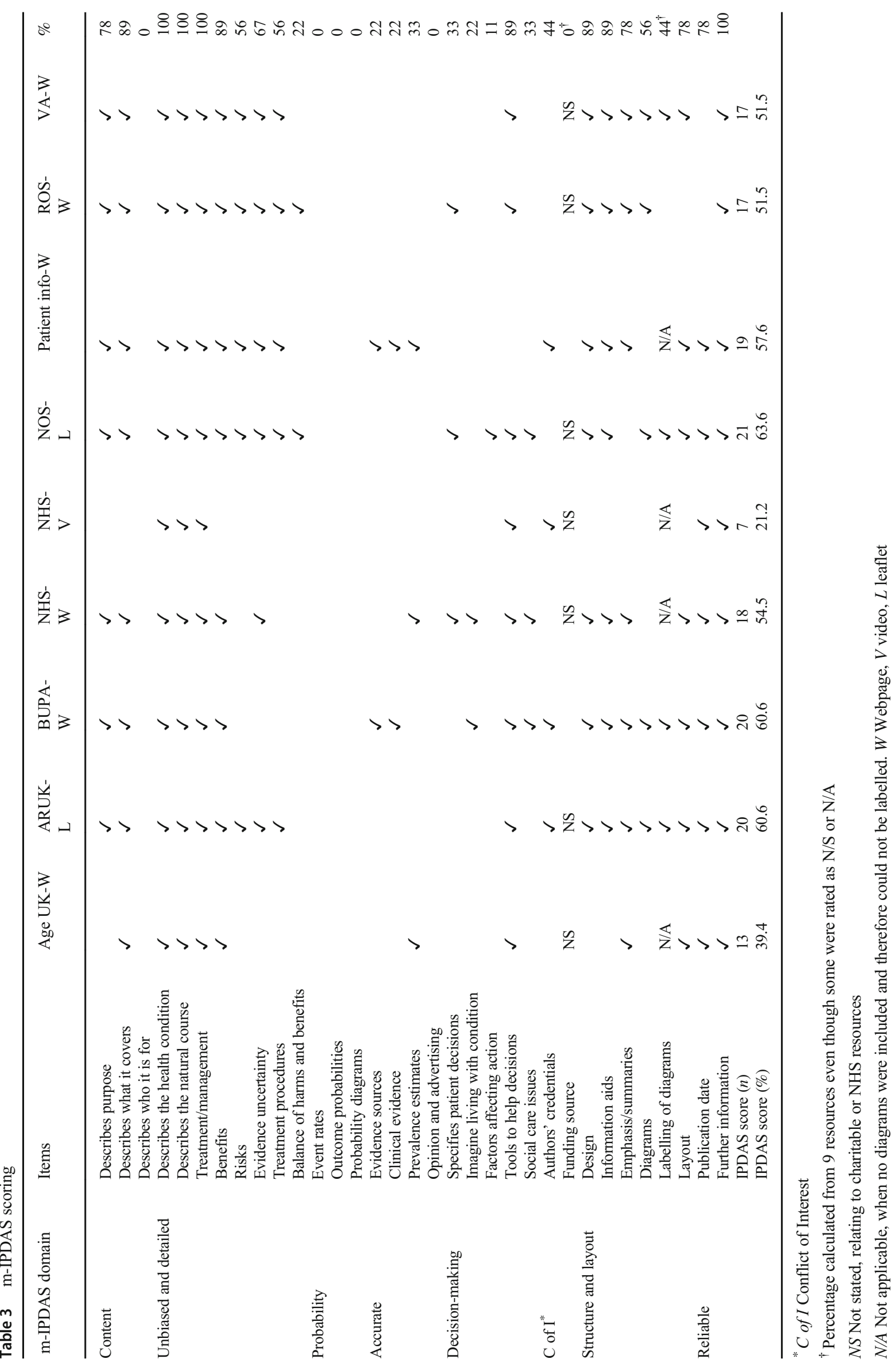


explicitly included a statement of who the information was targeted at. Although resources noted the benefits $(8 / 9$, $88 \%)$ and risks $(5 / 9,55 \%)$ of osteoporosis drugs, only two resources presented these in equal detail $(22 \%)$. While criteria relating to the description of the health condition and its natural course were met in all resources, in the resources that discussed drug treatment, five included discussion of the procedures for taking treatment. The use of consistent design and layout as well as information aids and emphasis, such as bullet points, noted in 7/9 (77\%) resources. In those that used diagrams $(5 / 9,55 \%)$, one did not provide any labels. All resources provided detail on further information, with $7 / 9(77 \%)$ providing a date of publication.

\section{Descriptive thematic analysis and stakeholder views}

Thirteen individual subthemes were identified relating to the five domains of Leventhal's Common-Sense Model; identity, cause, timeline, consequence and controllability (Fig. 2). Keywords and phrases from nine of these subthemes were discussed with the stakeholder group. The stakeholder group confirmed that the most commonly used resources had been included. A summary of the keywords and phrases identified within each domain and subtheme, is shown in Table 4, and described below. Key findings from the stakeholder discussion are presented alongside the thematic analysis results, to aid flow and reduce duplication of reporting.

\section{Identity}

Subthemes were identified relating to descriptions of osteoporosis, symptoms, fracture risk and explanation of bone density results. Although most resources explained osteoporosis meant bones were more likely to break more easily, descriptive terms used to describe the bone varied with terms such as 'spongy' [27, 28], 'weak' [27, 33], 'thin' [28, 32] and 'fragile'. Some of these terms were noted by the stakeholder group to be misleading (spongy) or liable to cause fear (fragile); the term 'weak' was preferred. Further, when resources used comparisons to 'average' to describe osteoporosis, such as 'bone density is lower than average' [33], this was viewed as confusing, as 'average' was not defined. One resource described 'idiopathic' and 'established' osteoporosis which was perceived by stakeholders as too technical for lay information [29].

Statements regarding symptoms were frequently contradictory. Osteoporosis was described to have either no symptoms [31-33] or 'often has no symptoms' [27]. Following a statement of no symptoms, one resource still went on to list 'signs that you can look out for' [31]. Of the resources that discussed general symptoms, [27, 28, 30-33] only two made it clear that all symptoms and signs were a result of indolent fractures [28, 30].

Fracture risk was described as risk or probability of breaking a bone over 10 years. Resources gave two different reasons as to why your fracture risk would be calculated to either inform treatment choices or inform the need for dual-energy $\mathrm{X}$-ray absorptiometry (DXA) scanning (a means of measuring bone mineral density), with two resources mentioning both $[27,28]$. Patient.info states that fracture risk calculations are used to 'determine your risk of developing osteoporosis' which was deemed misleading by the stakeholder group [32]. The stakeholder group expressed the view that it was important to explain that risk of fractures is influenced by a

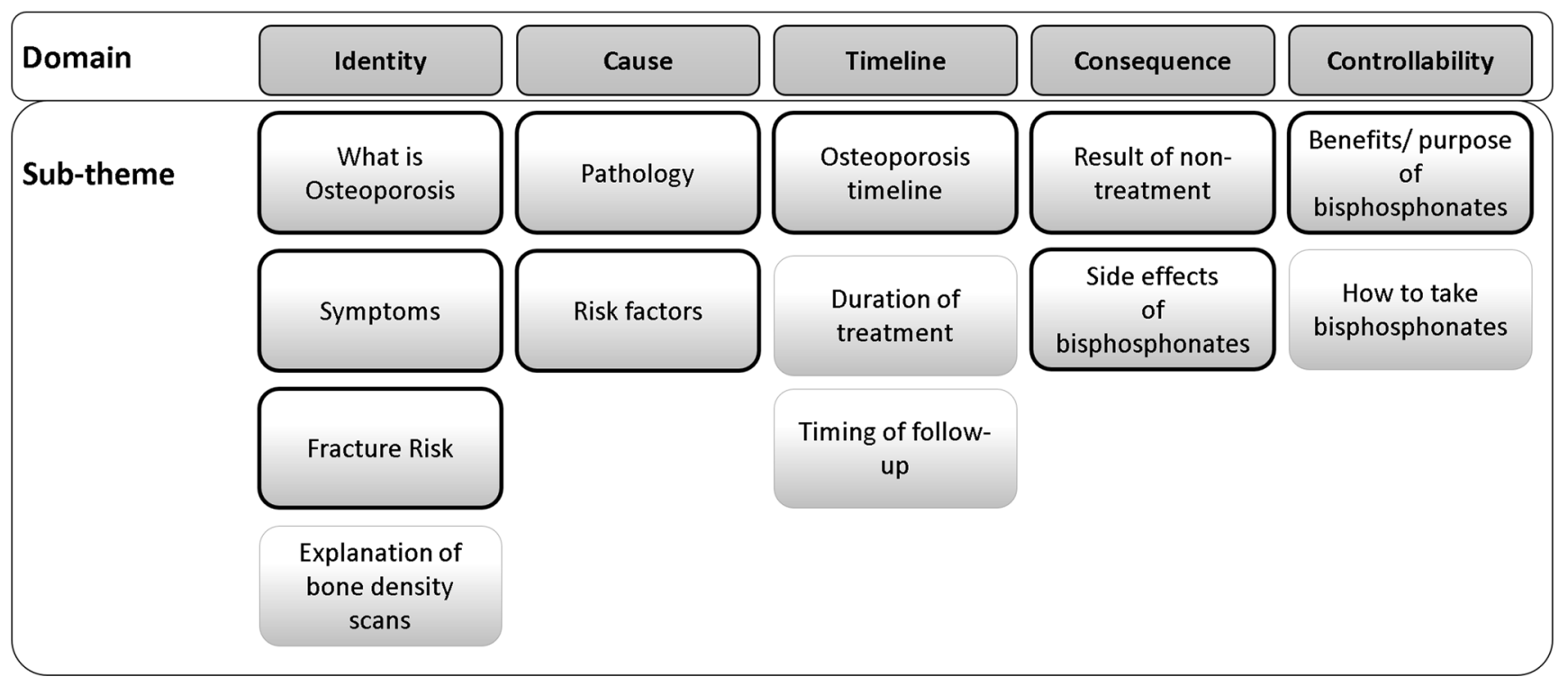

Fig. 2 Domains of Leventhal's common-sense model and subthemes of the narrative synthesis. Subthemes outlined in bold were discussed with the stakeholder group 


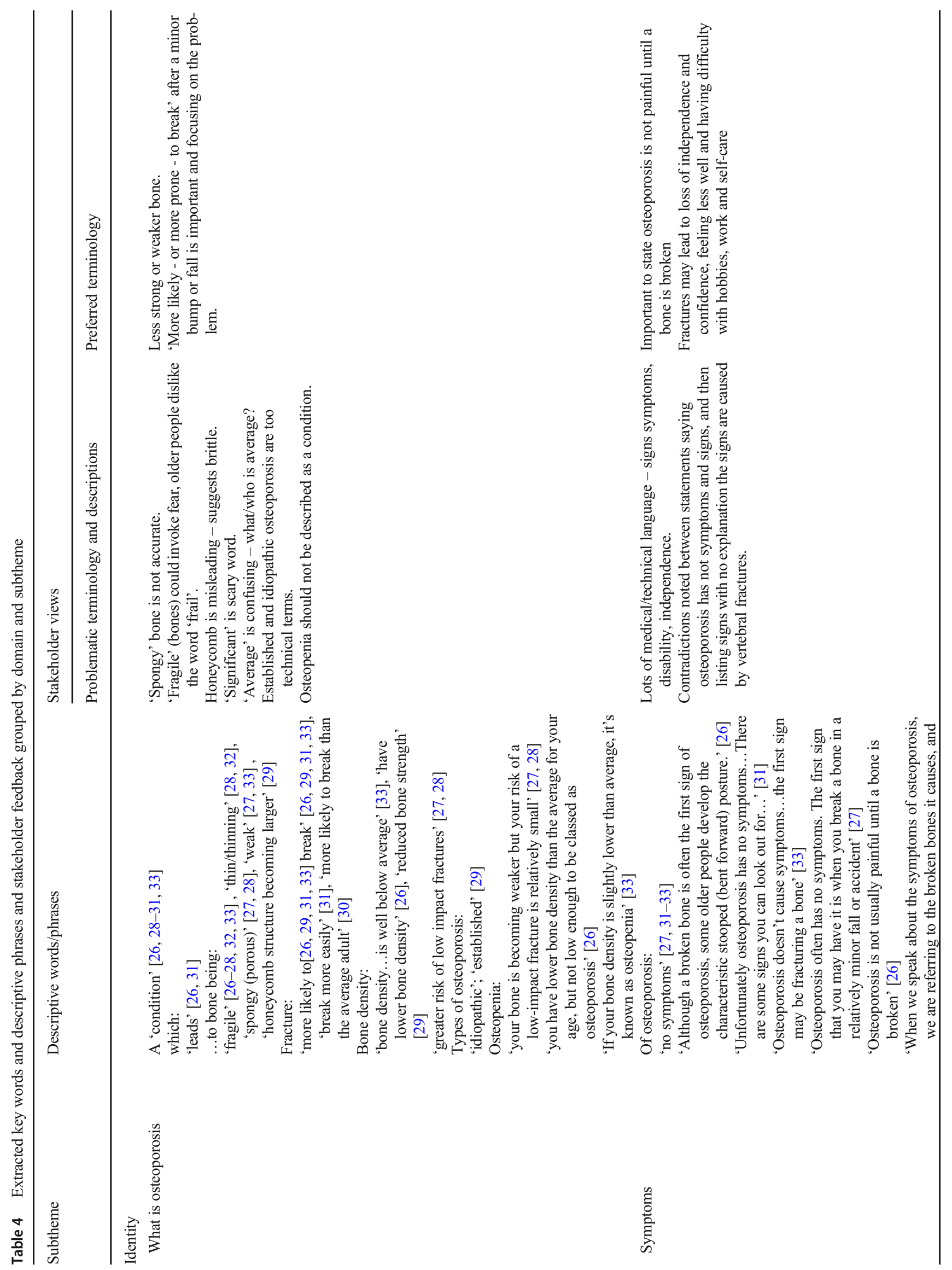




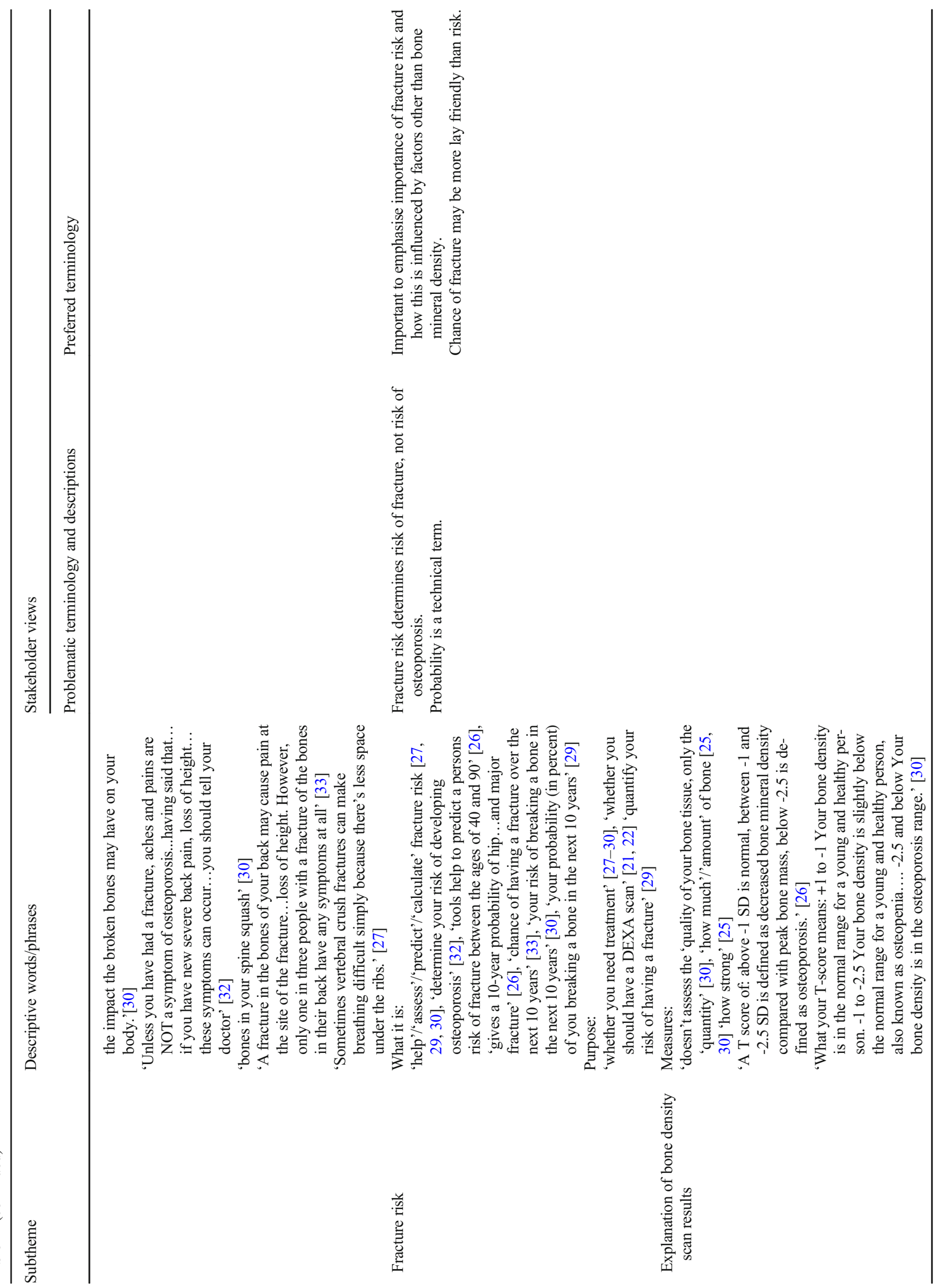




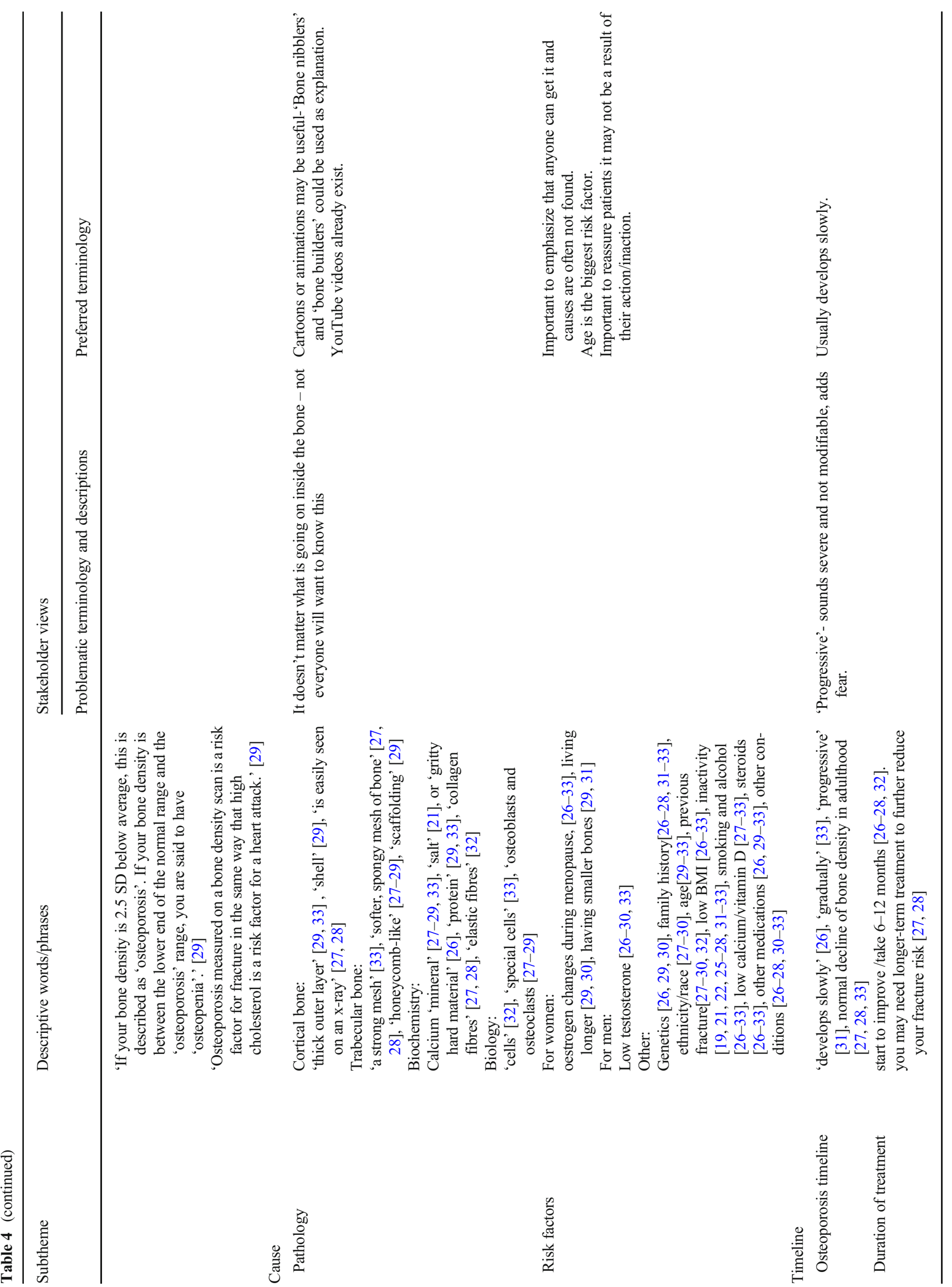




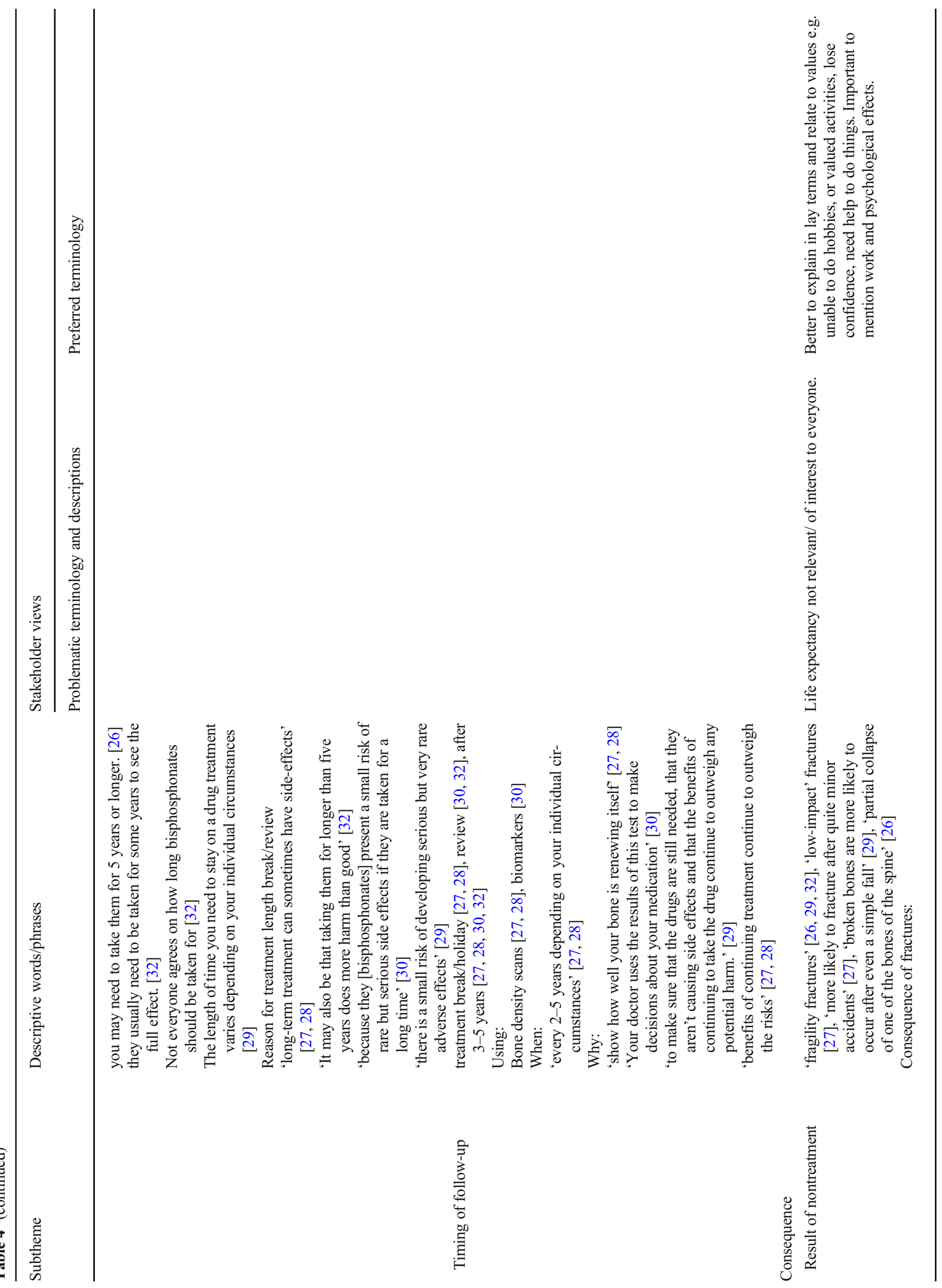



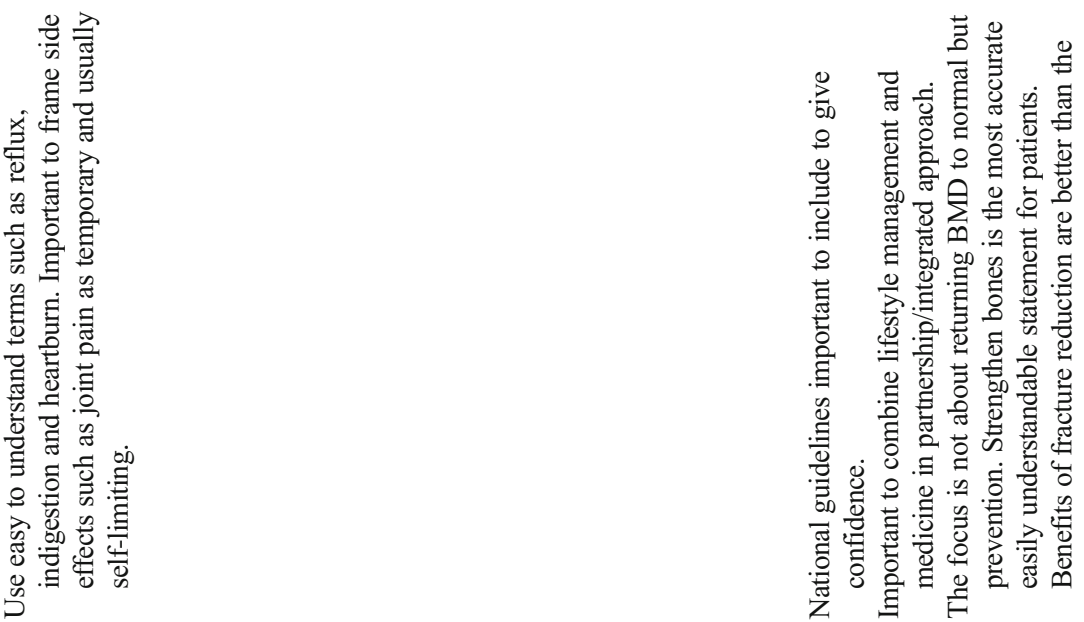

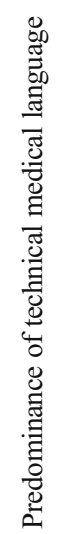

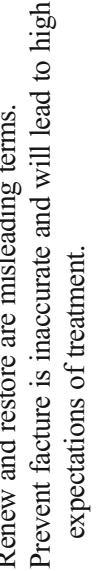

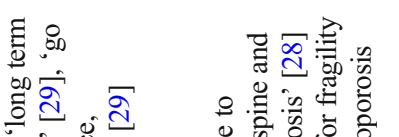
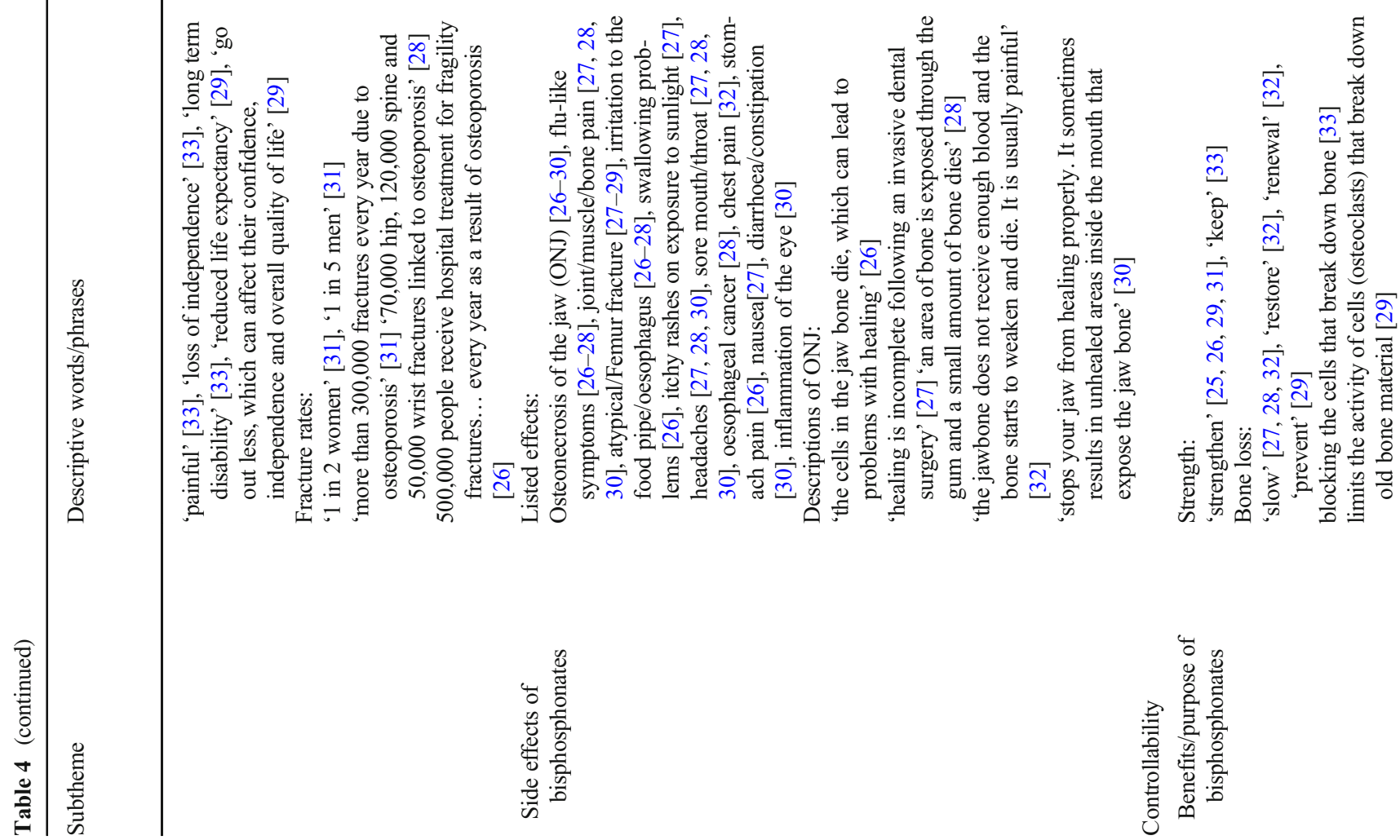
range of factors other than bone density alone and 'chance' was viewed as a more understandable term than 'risk' or 'probability'. Bone density was described as measuring quantity and strength. The NOS booklet additionally distinguished between density and strength, and why having low bone density does not automatically signify a reduction in strength [29].

\section{Causes}

Subthemes related to pathology and risk factors. Most resources included a description of bone histopathology including cortical and trabecular bone [27-29, 33], some went further to describe the biochemistry and cellular biology [27-29, 32, 33]; however, stakeholders noted that not everyone would want to know what is going on inside the bone, particularly when receiving information immediately after diagnosis. They did suggest that, for those people who were interested in biology, cartoons or animation might be helpful in explaining the cellular activity in simplified terms such as 'bone nibblers' vs 'bone builders'. Risk factors were variably described, but all resources mentioned common risk factors such as increasing age, steroids and early menopause. Stakeholders felt it was important to note that anyone can get osteoporosis, and that the causes are often not found; this was deemed important to avoid patients blaming themselves for poor lifestyle choices which may not have caused their osteoporosis.

\section{Timeline}

Subthemes identified related to the timeline of osteoporosis itself, duration of drug treatment and timing of follow-up. Loss of bone density over time was generally described as normal, however the term 'progressive' [31] was discussed as sounding severe and unmodifiable by stakeholders. Preference for the terms 'develops slowly' as used by the NHS website [26] was expressed. Treatment duration was discussed in relation to bisphosphonates, as would be expected, as these drugs are associated with increased risks with longer duration of treatment. Four resources indicated that bisphosphonates took 6-12 months to work [26-28, 32]. Timing of follow up was specified in four resources (3-5 years) [27-29, 32] and related to the possibility of a treatment break as 'long-term treatment can sometimes have side-effects' [27, 28]. The VA webpage outlined that the benefits of bisphosphonates are long term and would not be lost during a treatment break [27]. The NOS booklet also stated 'some may continue to have an effect for several years after treatment is stopped'; however it also stated 'drug treatments reduce 
the risk of breaking a bone most effectively while you are taking them' and 'when you stop treatment the benefit will start to wear off' [29].

\section{Consequences}

Subthemes identified related to consequences of osteoporosis and side effects of medication. In terms of consequences of osteoporosis, fractures were noted as sequels of both the condition and nontreatment. Fractures were described as 'fragility fractures' [26, 29, 32], 'low-impact' fractures [27] or fractures being caused by minor accidents/events $[29,33]$. The BUPA webpage and NOS booklet were the only resources to mention the psychosocial and physical consequences following a fracture such as long-term disability and reduced life expectancy $[29,33]$. Stakeholders noted that life expectancy may not be important to everyone, and that it would be better to relate fracture consequences to things important to patients using more lay terminology relating to work, hobbies and confidence.

In terms of side effects, osteonecrosis of the jaw (ONJ) and atypical femur fractures were described as 'less common' but 'more serious' [27, 30, 32] or 'rare' [26, 27, 32].

The ROS website used statements such as 'be reassured that it's unlikely you will experience any' [30] in relation to side effect risk, whilst Patient.info used phrases such as 'if you develop any symptoms....you should stop taking your tablets and see your doctor' [32]. Stakeholders noted that many of the terms used to describe side effects may not be easy to understand and suggested using more commonly understood language, e.g. gullet instead of oesophagus. Further, it was suggested that side-effect timelines be noted, such as in the case of joint pain with bisphosphonates which may be transient.

\section{Controllability}

Subthemes related to benefits of medication, and how to take medication. Generally, there was little information on benefits of drug treatment. Resources described benefits of bisphosphonate medication in terms bone strength, bone density and fracture risk. However, terminology varied, with the benefits described as 'reduced risk \{of fracture\}' [25, 26, 28, 34], 'prevent' \{fractures and bone loss\} [25, 27, 29, 31], 'increase' \{bone strength\} [27, 28], 'slow' \{bone loss\} [27, 28, 33]. Stakeholder discussions felt that terms relating to restoring, renewing or returning bone mineral density (BMD) to normal and preventing fracture were misleading. They recommended the term 'strengthen bone' and description of 'lowering the chance of future fracture'. Medication was also noted by stakeholders to give confidence - a psychosocial factor that could be included as a benefit.
Instructions around medication were given to 'ensure the medication is effectively absorbed', and that patients should 'carefully follow the instructions' [28] 'even though you won't be able to feel whether it's working' [27]. The sources gave a mixture of advice on instructions in relation to how much water to drink, how long to avoid food and how long to stay upright, with all resources suggesting more fluid or more time upright than the Summary of Product Characteristics for alendronic acid $70 \mathrm{mg}$, (which recommends one glass water and minimum 30 min respectively [35]). The NOS booklet added a tip to increase the likelihood of remembering to take the weekly tablet; 'the day your bins are emptied' [29].

\section{Discussion}

Good quality patient information can educate, inform and empower patients, enabling them to partake in shared decisionmaking regarding their conditions and support self-management. However, materials need to be of high quality and comprehensible in their presentation, language and content. Overall, this study found that patient information on osteoporosis had highly variable quality and scored poorly on readability assessments. The contradictions, inaccuracies and inconsistencies identified are particularly troubling, specifically relating to how symptoms are described, how to take medication and the benefits of treatment. We have produced 12 practical recommendations addressing the gaps identified by this study in order to support improvements in osteoporosis information.

Using readability as part of an assessment of quality, our findings suggest that all of the included online resources are too complex for the majority of UK residents. All resources in the current study scored above a grade 8 on the FKGL (except the NHS video on information describing osteoporosis drugs), which would exclude at least $43 \%$ of UK residents of working age, from understanding the information [12], and given, that health literacy declines with age [11] may exclude an even greater proportion of the population with, or at risk of osteoporosis.

Osteoporosis information resources are not alone in this, a study that included over 300 patient information resources available in doctors surgeries found that $75 \%$ were above the average national reading level [8]. Other studies focusing on individual topics such as urological conditions [36], trauma [37], and surgical reconstruction [38] have found similar results, with the majority of resources scoring above grade 8 on the FKGL.

The m-IPDAS quality assessment identified areas of weakness across the resources evaluated. Notably, resources scored poorly in the m-IPDAS domains of 'accuracy' and 'probabilities', with both the stakeholder discussion and the m-IPDAS highlighting an imbalance between the presentation of risks 
and benefits of bisphosphonates, in favour of the latter. This difference may, to some extent, be influenced by negative media on bisphosphonates [39]. Although benefits were discussed in less detail, the claims of benefits were sometimes misleading, suggesting fracture prevention and renewal of bone in line with a range of other conditions such as which has been described as overoptimistic [20]. Patients have previously expressed the desire to know the 'full picture'; however, they want it presented in a balanced manner with a delicate and truthful assessment of the risks and benefits [20]. It is also important patients have realistic expectations of treatment benefit, as poor alignment between outcome and expectations is likely to contribute to poor adherence. Research evidence suggests the inclusion of event rates and outcome probabilities, using simple frequencies, e.g. 1 in 100 , as a mechanism to facilitate balanced risk communication and shared decisionmaking [40]. However, as evidenced by the m-IPDAS, resources included event rates of fractures in general, but not probabilities relating to osteoporosis or specific osteoporotic fractures. To further ensure the relevance and appropriateness of the information, resources should explicitly state who the information was intended for. The m-IPDAS quality assessment identified that none of the included online osteoporosis resources provided such information. A disclosure of this kind is particularly pertinent for osteoporosis drug treatment, as drug information for females and males may differ, such as in the case of drug licensing [41], and our public contributor group also expressed the importance of stating whether resources were generally relevant to both men and women. Only two resources reference their evidence, as has previously been seen in other information sources for other conditions, with a high proportion failing to disclose their evidence sources [5]. Disclosure of funding is also important, especially for a subspeciality which has been heavily criticised for its connections with the pharmaceutical industry [42].

Our thematic analysis revealed a range of different descriptors for the condition; the term osteoporosis has different meanings (e.g. low bone mineral density, previous fragility fracture), and these different representations may contribute to confusion, e.g. about the relationship with symptoms [43]. To address confusion and inconsistency, the stakeholder group supported the use of simplified, clear terminology and an avoidance of complex science in their suggestions regarding osteoporosis description, medication benefits and side effects. Stakeholders suggested that the benefits of osteoporosis drug treatment should be made relatable to patient's values, motivations and priorities, such as hobbies and confidence levels. When highlighting risks, resources should focus on the potential social, psychological and physical consequences of not taking osteoporosis treatment (e.g. care home admission, reduced independence), as these factors scored low in the m-IPDAS quality assessment. This information should be linked to the benefits of treatment; previous research has highlighted that people with osteoporosis did not understand the purpose of drug treatment [13] and low perceived need for treatment is associated with nonadherence [44].

\section{Recommendations for improving patient information about osteoporosis}

\section{General considerations:}

1. Explicitly state the target audience for the information

2. Simplify language used, avoid technical language and ensure readability is appropriate

3. Cite sources of evidence

4. Cite sources of funding

\section{When describing osteoporosis:}

5. Use recommended terms, e.g. 'weak bone' and avoid terms such as 'spongy' and 'honeycomb'

6. Explain osteoporosis develops slowly and anyone can get it

7. Avoid contradictory statements about absence or presence of symptoms and ensure symptoms and signs are explained as caused by fractures

8. Include discussion of the physical, social and psychological impact of osteoporotic fractures, but avoid descriptions that may induce fear

9. Use labelled images or animations where possible, e.g. to illustrate pathology

\section{When describing osteoporosis drug treatment:}

10. Balance the amount of information about benefits and risks of drug treatment, and describe using probabilities or simple event rates

11. Describe the benefits of treatment as strengthening bone and lowering the chance of future fracture, and avoid misleading terms such as 'prevent', 'renew' and 'restore'

12. Provide accurate information about how to take bisphosphonates, and how long for, and explain why these procedures are important

Given the need for clear communication of the benefits versus the risks of drug treatment is internationally recognised as a priority area in improving osteoporosis management and care $[9,45]$, we recommend that providers of osteoporosis health care information assess their current resources against these criteria to identify avenues for improvement that will make patient information more accessible and readable. 
Furthermore, existing providers need to address any uncertainty and confusion in areas relating to the nature of the condition, medication, and controllability [13]. When producing new resources, we suggest providers utilise these recommendations to check that they align accordingly to increase consistency, and ensure high quality and readability of osteoporosis health information.

\section{Strength and weaknesses}

Multiple methods were utilised in this study to explore online patient information about osteoporosis, including an in-depth readability and quality assessment (utilising the m-IPDAS); a descriptive thematic analysis informed by theory; and engagement with a diverse stakeholder group consisting of experts, clinicians and patients. Including the views of public contributors is essential in design and appraisal of patient information to inform relevance, understanding and acceptability of information $[13,20]$. Each method contributed to a robust and rounded understanding of the quality of osteoporosis patient information, and we believe these methods would be of use to study patient information about other subjects.

A number of limitations are worthy of mention. First, information about lifestyle was not included in this investigation, and therefore, the quality of information about nonpharmacological management remains to be determined. We focused upon the quality and readability of information about osteoporosis and osteoporosis drug treatments to inform the design of the iFraP intervention and also based on two of the three top priorities in osteoporosis research: having easy access to information and understanding further the safety and benefit of osteoporosis drug treatment [2]. Second, readability scores, utilised in this study as a quality indicator, have been criticised for use in medical situations, as they cannot account for necessary medical terms [8]. However, previous studies have shown good reproducibility, correlations with other readability scales and have been previously utilised within a health information context [11,36, 37].

Third, it is likely that quality and readability assessments were related to the length of the text included, and we did not formally evaluate this relationship. For example, the video, the shortest resource scored best on readability and worst on m-IPDAS. The longest resource (NOS booklet), also scored highest in the m-IPDAS. High scores in m-IPDAS did not necessarily relate to quality overall, with a number of challenging or confusing textual descriptors identified by stakeholders, emphasizing the value of the combined methods used. Furthermore, we did make selections on which text to include: some of the providers of information do provide a wide range of resources and may have included relevant patient information or content in other resources or documents that we did not include. However, where relevant e.g. disclosure of funding, we did attempt to find information on the source webpage. Finally, although visual elements (e.g. use of images) are included within the IPDAS rating, our study did not include a detailed evaluation of the style, layout or format of resources, which remains in need of further study.

\section{Conclusion}

This study found that commonly accessed patient information resources on osteoporosis were too complex for most of the population and identified areas of weakness using a validated quality measure. Inconsistencies and inaccuracies were noted in the phrases used as well as terms that were difficult for patients to understand. We have produced practical recommendations for information providers to support improvements in understanding, relevance, balance and bias, and to address information gaps.

Supplementary Information The online version contains supplementary material available at https://doi.org/10.1007/s00198-020-05800-7.

Funding This study was funded by the National Institute for Health Research (NIHR) [Clinician Scientist Award (CS-2018-18-ST2-010)/ NIHR Academy], the Royal Osteoporosis Society, and the Haywood Rheumatology Research and Development Foundation. ZP is funded by the NIHR, Clinician Scientist Award (CS-2018-18-ST2-010)/NIHR Academy. FCM is part funded by the NIHR Clinical Research Network Scholar Programme. AH is a NIHR funded Academic Clinical Fellow. CJ is part funded by the NIHR Applied Research Collaboration West Midlands.

Data availability The data (patient information websites and booklets) are freely available.

\section{Compliance with ethical standards}

Conflicts of interest Sarah Leyland was involved in the development of the ROS and NHS resources evaluated in this paper. Fay CrawfordManning, Charlotte Greenhall, Ashley Hawarden, Laurna Bullock, Clare Jinks, Joanne Protheroe and Zoe Paskins declare that they have no conflict of interest. The views expressed are those of the authors and not necessarily those of the NHS, the NIHR or the Department of Health \& Social Care.

Code availability Not applicable.

Open Access This article is licensed under a Creative Commons Attribution-NonCommercial 4.0 International License, which permits any non-commercial use, sharing, adaptation, distribution and reproduction in any medium or format, as long as you give appropriate credit to the original author(s) and the source, provide a link to the Creative Commons licence, and indicate if changes were made. The images or other third party material in this article are included in the article's Creative Commons licence, unless indicated otherwise in a credit line to the material. If material is not included in the article's Creative Commons licence and your intended use is not permitted by statutory regulation or exceeds the permitted use, you will need to obtain permission directly from the copyright holder. To view a copy of this licence, visit http:// creativecommons.org/licenses/by-nc/4.0/. 


\section{References}

1. Vahdat S, Hamzehgardeshi L, Hessam S, Hamzehgardeshi Z (2014) Patient involvement in health care decision making: a review. Iran Red Crescent Med J:16. https://doi.org/10.5812/ircmj. 12454

2. Paskins Z, Jinks C, Mahmood W, Jayakumar P, Sangan CB, Belcher J, Gwilym S (2017) Public priorities for osteoporosis and fracture research: results from a general population survey. Arch Osteoporos 12:45. https://doi.org/10.1007/s11657-017-0340-5

3. Fox S, Duggan M (2013) Health Online:2013

4. Flash Eurobarometer 4 (2014) European citizens' digital health literacy. European Comission

5. Ferreira G, Traeger AC, Machado G, O'Keeffe M, Maher CG (2019) Credibility, accuracy, and comprehensiveness of internetbased information about low back pain: a systematic review. J Med Internet Res 21:e13357. https://doi.org/10.2196/13357

6. Coulter A (1998) Evidence based patient information. BMJ 317: 225-226. https://doi.org/10.1136/bmj.317.7153.225

7. Montori VM, LeBlanc A, Buchholz A, Stilwell DL, Tsapas A (2013) Basing information on comprehensive, critically appraised, and up-to-date syntheses of the scientific evidence: a quality dimension of the International Patient Decision Aid Standards. BMC Med Inform Decis Mak 13(2):1-7

8. Protheroe J, Estacio EV, Saidy-Khan S (2015) Patient information materials in general practices and promotion of health literacy: an observational study of their effectiveness. Br J Gen Pract 65:e192e197. https://doi.org/10.3399/bjgp15X684013

9. Khosla S, Shane E (2016) A crisis in the treatment of osteoporosis. J Bone Miner Res 31:1485-1487. https://doi.org/10.1002/jbmr.2888

10. Selden CR, Zorn M, Ratzan SC, Parker RM (2000) Health literacy. Current bibliographies in medicine

11. Harding C, Romanou E, Williams J, Peters M (2012) The 2011 skills for life survey: a survey of literacy, numeracy, and ICT levels in England. Department for Business, Innovation and Skills

12. Rowlands G, Protheroe J, Winkley J, Richardson M, Seed PT, Rudd R (2015) A mismatch between population health literacy and the complexity of health information: an observational study. Br J Gen Pract 65:e379-e386. https://doi.org/10.3399/ bjgp15X685285

13. Raybould G, Babatunde O, Evans AL, Jordan JL, Paskins Z (2018) Expressed information needs of patients with osteoporosis and/or fragility fractures: a systematic review. Arch Osteoporos 13:55. https://doi.org/10.1007/s11657-018-0470-4

14. Braun V, Clarke V (2006) Using thematic analysis in psychology. Qual Res Psychol 3:77-101. https://doi.org/10.1191/ 1478088706qp063oa

15. Grime JC, Ong BN (2007) Constructing osteoarthritis through discourse - a qualitative analysis of six patient information leaflets on osteoarthritis. BMC Musculoskelet Disord 8:34. https://doi.org/10. 1186/1471-2474-8-34

16. Misra P, Kasabwala K, Agarwal N, Eloy JA, Liu JK (2012) Readability analysis of internet-based patient information regarding skull base tumors. J Neuro-Oncol 109:573-580

17. Flesch R (1948) A new readability yardstick. J Appl Psychol 32: 221-233. https://doi.org/10.1037/h0057532

18. Kincaid J, Fishburne R, Rogers R, Chissom B (1975) Derivation Of new readability formulas (Automated Readability Index, Fog Count And Flesch Reading Ease Formula) for navy enlisted personnel. Institute for Simulation and Training

19. Rolland P (2016) Reading level of drug information printouts: a barrier to effective communication of patient medication information: Drug Information Journal. https://doi.org/10.1177/ 009286150003400440
20. Coulter A, Entwistle V, Gilbert D (1999) Sharing decisions with patients: is the information good enough? BMJ 318:318-322. https://doi.org/10.1136/bmj.318.7179.318

21. Elwyn G, O'Connor AM, Bennett C, Newcombe RG, Politi M, Durand M, Drake E, Joseph-Williams N, Khangura S, Saarimaki A, Sivell S, Stiel M, Bernstein SJ, Col N, Coulter A, Eden K, Härter M, Rovner MH, Moumjid N, Stacey D, Thomson R, Whelan T, van der Weijden T, Edwards A (2009) Assessing the quality of decision support technologies using the International Patient Decision Aid Standards instrument (IPDASi). PLoS One 4:e4705. https://doi.org/ 10.1371/journal.pone.0004705

22. Leventhal H, Benyamini Y, Brownlee S, Diefenbach M, Leventhal EA, Patrick-Miller L, Robitaille C (1997) Illness representations: theoretical foundations, In Anonymous (1997) Perceptions of health and illness: Current research and applications. Harwood Academic Publishers, Amsterdam, pp 19-45

23. Horne R, Cooper V, Wileman V, Chan A (2019) Supporting adherence to medicines for long-term conditions: a perceptions and practicalities approach based on an extended common-sense model. Eur Psychol 24:82-96. https://doi.org/10.1027/1016-9040/ a000353

24. Shiyanbola OO, Ward EC, Brown CM (2018) Utilizing the common sense model to explore African Americans' perception of type 2 diabetes: a qualitative study. PLoS One 13:e0207692

25. Leyland S (2017) Osteoporosis video (NHS). Date Accessed: $20^{\text {th }}$ December 2019. https://www.versusarthritis.org/about-arthritis/ conditions/osteoporosis/

26. National Health Service (2019) Osteoporosis (NHS). Date Accessed: $20^{\text {th }}$ December 2019. https://www.nhs.uk/conditions/ osteoporosis/

27. Versus Arthritis (Osteoporosis (VA). Date Accessed: $20^{\text {th }}$ December 2019. https://www.versusarthritis.org/about-arthritis/ conditions/osteoporosis/

28. Palferman T (2016) Osteoporosis (Arthritis Research, UK). Date Accessed: $20^{\text {th }}$ December 2019

29. National Osteoporosis Society (2016) All about Osteoporosis and bone health (NOS). Date Accessed: $20^{\text {th }}$ December 2019

30. Royal Osteoporosis Society ( Understanding Osteoporosis (ROS). Date Accessed: $20^{\text {th }}$ December 2019. https://theros.org.uk/ information-and-support/osteoporosis

31. Age UK (2019) Osteoporosis (Age UK). Date Accessed: $20^{\text {th }}$ December 2019. https://www.ageuk.org.uk/information-advice/ health-wellbeing/conditions-illnesses/osteoporosis

32. Harding M, Cox J Osteoporosis (Patient-info). Date Accessed: $20^{\text {th }}$ December 2019. https://patient.info/bones-joints-muscles/ osteoporosis-leaflet

33. Bhalara S, Routh K (2019) Osteoporosis (BUPA). Date Accessed: $20^{\text {th }}$ December 2019. https://www.bupa.co.uk/health-information/ muscles-bones-joints/osteoporosis

34. Versus Arthritis (2018) A research roadmap for pain. https://www. versusarthritis.org/media/1672/research-roadmap-pain.pdf

35. Accord Healthcare Limited (2017) Alendronic acid once weekly $70 \mathrm{mg}$ tablets. 2020

36. Dalziel K, Leveridge MJ, Steele SS, Izard JP (2016) An analysis of the readability of patient information materials for common urological conditions. Can Urol Assoc J 10:167-170. https://doi.org/10. 5489/cuaj.3578

37. Eltorai AEM, Ghanian S, Adams CA, Born CT, Daniels AH (2014) Readability of patient education materials on the American Association for Surgery of Trauma Website. Arch Trauma Res 3. https://doi.org/10.5812/atr.18161

38. Polishchuk DL, Hashem J, Sabharwal S (2012) Readability of online patient education materials on adult reconstruction web sites. J Arthroplast 27:716-719. https://doi.org/10.1016/j.arth.2011.08.020

39. Merle B, Haesebaert J, Bedouet A, Barraud L, Flori M, Schott A, Dupraz C (2019) Osteoporosis prevention: where are the barriers to 
improvement in French general practitioners? A qualitative study. PLoS One 14:e0219681. https://doi.org/10.1371/journal.pone. 0219681

40. Stacey D, Légaré F, Col NF, Bennett CL, Barry MJ, Eden KB, Holmes-Rovner M, Llewellyn-Thomas H, Lyddiatt A, Thomson R, Trevena L, Wu JHC (2014) Decision aids for people facing health treatment or screening decisions. Cochrane Database Syst Rev:CD001431. https://doi.org/10.1002/14651858.CD001431. pub4

41. National Osteoporosis Guideline Group (2017) NOGG 2017: clinical guideline for the prevention and treatment of osteoporosis

42. Järvinen TL, Michaëlsson K, Jokihaara J, Collins GS, Perry TL, Mintzes B, Musini V, Erviti J, Gorricho J, Wright JM, Sievänen H (2015) Overdiagnosis of bone fragility in the quest to prevent hip fracture. BMJ 350:h2088. https://doi.org/10.1136/bmj.h2088
43. Paskins Z, Ong T, Armstrong DJ (2020) Bringing osteoporosis up to date: time to address the identity crisis. Age Ageing 49:329-331. https://doi.org/10.1093/ageing/afaa022

44. Horne R, Chapman SCE, Parham R, Freemantle N, Forbes A, Cooper V (2013) Understanding patients' adherence-related beliefs about medicines prescribed for long-term conditions: a metaanalytic review of the necessity-concerns framework. PLoS One 8:e80633. https://doi.org/10.1371/journal.pone.0080633

45. Harvey NC, McCloskey EV (2016) Gaps and solutions in bone health. A Global Framework for Improvement. International Osteoporosis Foundation

Publisher's note Springer Nature remains neutral with regard to jurisdictional claims in published maps and institutional affiliations. 